\title{
LES STADES LARVAIRES DE MEIOGYMNOPHALLUS STRIGATUS \\ (M. V. LEBOUR, 1908) n. comb. (Trematoda : Gymnophallidae)
}

\author{
P. BARTOLI*
}

RÉSUMÉ. Les cercaires et les métacercaires de Meiogymnophallus strigatus (M. V. Lebour, 1908) n. comb. ont été trouvées en quelques points des côtes de Camargue (France méridionale). Les sporocystes et les cercaires parasitent Tellina tenuis tandis que les métacercaires se rencontrent dans plusieurs espèces de Lamellibranches, principalement dans Donax trunculus et D. semistriatus. Les cercaires et les métacercaires sont décrites. La filiation entre ces deux stades larvaires est expérimentalement démontrée. Le microhabitat des métacercaires est étudié ; les réactions de D. trunculus vis-à-vis du parasite sont précisées. Deux catégories de métacercaires sont mises en évidence : celles vivant librement dans l'espace extrapalléal (elles ont une maturité génitale avancée) et celles qui sont circonscrites par les expansions palléales de l'hôte (elles ont une vessie excrétrice énorme). Le stade adulte n'a pu être obtenu, ni par la voie de l'expérimentation, ni dans la nature. L'attribution de Cercaria strigata M. V. Lebour, 1908 (Cercaria n'est pas un nom générique) au genre Meiogymnophallus $\mathrm{H}$. L. Ching, 1965 repose sur l'étude détaillée de l'anatomie des métacercaires âgées.

\section{The larval stages of Meiogymnophallus strigatus (M. V. Lebour, 1908) n. comb. (Trematoda : Gymnophallidae).}

SUMMARY. Cercariae and metacercariae of Meiogymnophallus strigatus (M. V. Lebour, 1908) have been found in some places along the coast of Camargue (South of France). Sporocysts and cercariae are parasites of Tellina tenuis. Metacercariae occur in various Bivalves, mainly in Donax trunculus and D. semistriatus. Cercariae and metacercariae are described. Relationship between cercariae and metacercariae is demonstrated. Metacercarial microhabitat is delimited. Reaction of D. trunculus in front of the metacercarial infection is studied. Some metacercariae are freeliving in the extrapallial space, between the mantle and the shell. These larvae have an advanced genital maturity. Most of the metacercariae are massed together and are enclosed by the host's epithelium. Their excretory vesicle, which is packed with blackish granules, almost fills the entire body. Attempts to infect laboratory reared birds by feeding metacercariae from Donax trunculus were not successful. The natural final host is unknown. In consideration of the metacercarial anatomy, Cercaria strigata M. V. Lebour, 1908 is placed in the genus Meiogymnophallus H. L. Ching, 1965.

* Laboratoire de Zoologie Marine, Faculté des Sciences et Techniques de Saint-Jérôme, Université d'AixMarseille III, F 13397 Marseille Cedex 13.

Accepté le 23 décembre 1982 . 
Cercaria strigata M. V. Lebour, 1908 est un Digène parasite de Lamellibranches des genres Donax et Tellina. Décrit à l'origine comme une cercaire, cet Helminthe s'est révélé être en fait une métacercaire. Ces métacercaires paraissent avoir été signalées pour la première fois par A. Giard (1897) à Boulogne-sur-mer dans Donax trunculus L., Tellina fabula Gronov, Tellina tenuis Da Costa et Tellina solidula (=T. balthica L., ex parte). P. Pelseneer (1906) a été le premier à donner une illustration de ces formes larvaires que l'on " rencontre fréquemment entre le manteau et la coquille de Donax et de divers autres Lamellibranches ". En 1907, A. Giard précise les dimensions de ce parasite auquel M. V. Lebour (1908) donne le nom de Cercaria strigata. En 1912, R. P. Dollfus redécrit Cercaria strigata en précisant qu'il s'agit là d'une métacercaire et non d'une cercaire. En 1939, G. Rees décrit à nouveau Cercaria strigata et indique avoir trouvé les cercaires correspondantes dans Cardium edule. Toutefois ces dernières ne sont pas décrites et la filiation cercaire-métacercaire n'est pas démontrée. Depuis cette date, il n'a plus été fait mention de cette espèce.

Nous avons retrouvé ce parasite dans plusieurs espèces de Lamellibranches de la biocénose des Sables Fins Superficiels (S.F.S.) des côtes de Camargue (France méridionale). A deux reprises nous avons trouvé les cercaires correspondantes. Grâce aux infestations expérimentales de Lamellibranches sains, nous avons obtenu les métacercaires. La filiation cercaire-métacercaire est ainsi démontrée. Malheureusement, toutes les tentatives pour obtenir les adultes chez les Oiseaux ont échoué. Cependant, sur la base de l'anatomie de la métacercaire âgée, nous pensons pouvoir attribuer cette espèce au genre Meiogymnophallus H. L. Ching, 1965. Ainsi, Cercaria strigata M. V. Lebour, 1908 devient Meiogymnophallus strigatus (M. V. Lebour, 1908) n. comb. La conséquence immédiate de cette nouvelle combinaison est l'uniformisation d'une nomenclature désordonnée. En effet, les différents auteurs ont parlé successivement de Cercaria strigata, de "Cercaria "strigata, de Metacercaria strigata, de Gymnophallus somateriae var. strigatus et de Metacercaria (Gymnophallus) strigata.

\section{I. - Les sporocystes}

Premier hôte intermédiaire: Tellina tenuis Da Costa.

Prévalence: deux individus parasités sur un total de 3260 Tellina tenuis examinés en neuf années de recherche, soit une prévalence de $0,05 \%$.

Microhabitats : glande digestive et gonade. Il y a castration parasitaire.

Dimensions : les dimensions $\left({ }^{*}\right)$ sont celles de 20 sporocystes montés en préparation.

$533-853(724) \times 133-213(170)$

Forme: ce sont des formations allongées, arrondies à une extrémité, effilées à l'autre (fig. 1).

* Les dimensions sont exprimées en microns $(\mu \mathrm{m})$; elles se rapportent à des animaux fixés entre lame et lamelle, modérément comprimés et montés en préparation. Le premier chiffre indique la taille minimale, le second les dimensions maximales, celui entre parenthèses la valeur moyenne. 


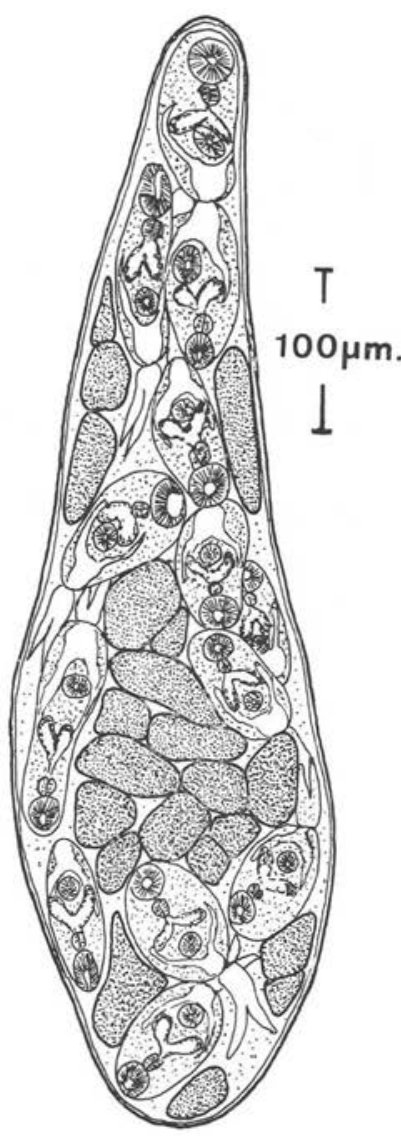

FIG. I. - Meiogymnophallus strigatus (M. V. Lebour, 1908) n. comb. Sporocyste. Glande digestive de Tellina tenuis. Camargue.

\section{II. - Les cercaires}

Dans la description qui suit, seules ont été prises en considération les cercaires émises spontanément par le premier hôte intermédiaire.

Les cercaires appartiennent au type furcocerque (fig. $2 \mathrm{~A}$ ); la forme générale du corps est ovale.

Dimensions.

Les dimensions sont celles de 20 individus montés en préparation.

Longueur du corps : 112-150 (136).

Largeur du corps : 59-95 (73).

Ventouse orale : $30-38(34) \times 30-43(36)$. 

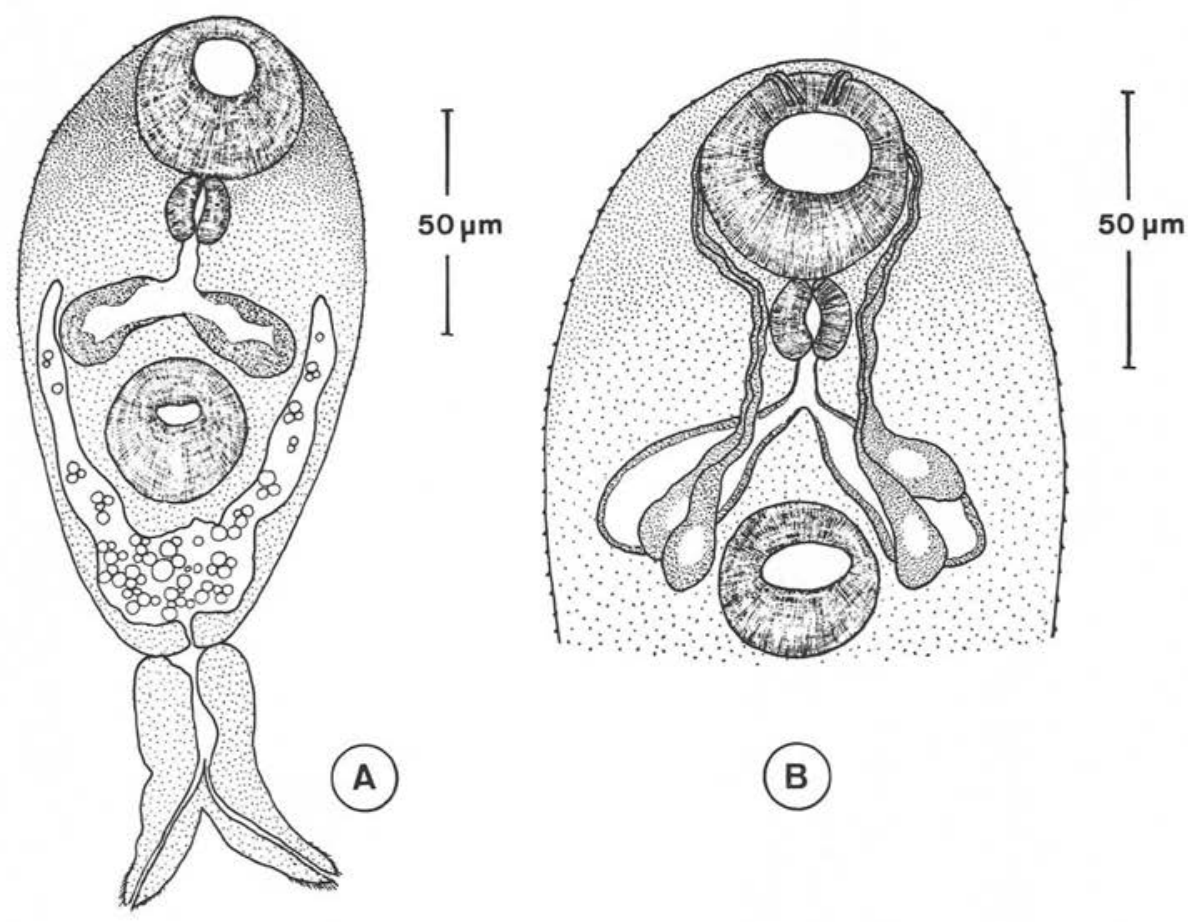

FIG. 2. - Meiogymnophallus strigatus (M. V. Lebour, I908) n. comb. Cercaire. Premier hôte intermédiaire : Tellina tenuis. Camargue.

A : morphologie générale ; $\mathrm{B}$ : glandes de pénétration.

Ventouse ventrale : $20-32(28) \times 28-41(31)$.

Rapport ventousaire, VO/VV : 1,07-1,50 $(1,23)$

Espace préacétabulaire : 62-86 (75).

Espace postacétabulaire : 19-46 (31).

Pharynx : 13-17 (15) × 12-20 (16).

Rapport ventouse orale-pharynx, $\mathrm{VO} / \mathrm{P}=1,88-2,57(2,28)$.

Esophage : 6-25 (17).

Queue : tronc : $30-75(45) \times 9-27(20)$.

fourchons : 28-45 (37) $\times$ 9-17 (12).

\section{Tégument.}

Le corps est revêtu d'épines extrêmement petites.

\section{Glandes de pénétration.}

Les glandes de pénétration sont au nombre de deux paires ; leur corps cellulaire est localisé près des caecums digestifs (fig. 2 B). De chaque côté, les canalicules 
cheminent parallèlement l'un à l'autre, longent le pharynx, passent au-dessus de la ventouse orale et s'ouvrent à l'extérieur sur la lèvre antérieure de celle-ci.

\section{Ventouses.}

La ventouse orale est terminale. La ventouse ventrale, légèrement aplatie, est disposée approximativement à la limite des tiers moyen et postérieur. Les six boutons sensoriels du cycle acétabulaire externe sont bien distincts.

\section{Appareil digestif.}

Un prépharynx est à peine distinct; le pharynx est globuleux, l'œsophage peu important. Les caecums digestifs sont composés chacun de six à sept cellules épithéliales ; leur extrémité postérieure atteint parfois le centre de la ventouse ventrale.

\section{Appareil génital.}

L’appareil génital n'est pas ébauché.

\section{Appareil excréteur.}

Aucune flamme vibratile ni aucun canalicule excréteur ne peuvent être distingués dans le corps de la cercaire; seule la boucle du gros canal cilié est visible. La vessie excrétrice est constituée par un tronc volumineux et par deux branches embrassant les caecums digestifs; ces dernières s'étendent jusqu'au niveau de l'œsophage. La vessie est remplie d'un petit nombre de granules d'excrétion ; leur diamètre ne dépasse pas $3 \mu \mathrm{m}$.

\section{Quene.}

La queue est revêtue d'épines sur toute sa surface ; l'extrémité de chaque fourchon est garnie de nombreux petits poils. La queue est dépourvue de protonéphridies mais elle est parcourue par le canal excréteur, prolongement de la vessie ; après s'être divisé, chaque canalicule débouche à l'extrémité d'un fourchon.

\section{III. - Les métacercaires}

\section{1 - Les deuxièmes hôtes intermédiaires naturels.}

Presque tous les deuxièmes hôtes intermédiaires naturels font partie de la biocénose des Sables Fins Superficiels (S.F.S.) définie par J. M. Pérès et J. Picard (1964).

\section{Donacidae : Donax trunculus L.}

Donax semistriatus Poli.

Corbulidae : Lentidium mediterraneum (Costa).

Solenidae : Solen marginatus Pennant.

Ensis ensis (L.).

Pharus legumen (L.).

Mactridae : Spisula subtruncata (Da Costa).

Lucinidae : Loripes lacteus (L.). 


\section{2 - Prévalence.}

La prévalence est d'autant plus élevée que l'hydrodynamisme est peu important. Dans certaines stations bien protégées, comme par exemple le fond du golfe des Saintes-Maries-de-la-Mer, elle peut atteindre $100 \%$.

\section{3 - Microhabitat.}

Les métacercaires de Meiogymnophallus strigatus ne sont jamais enkystées ; elles sont localisées entre le manteau et la coquille de leurs divers deuxièmes hôtes. Les parasites ne mènent pas une existence active à l'intérieur de l'espace extrapalléal mais vivent fixés à la surface externe du manteau.

On observe quelquefois des métacercaires solitaires, éparses à la surface du pallium. Au contact des parasites le manteau réagit sous la forme de villosités palléales plus ou moins importantes. C'est parmi ces expansions que les larves trouvent refuge. Les métacercaires ne sont pas englobées dans des vésicules; elles demeurent largement en relation avec l'espace extrapalléal général.

En dehors de ces rares métacercaires solitaires, la plus grande partie des parasites est localisée presque exclusivement au niveau de la région dorsale de chaque lobe palléal, dans la région cardiaque. Au contact des nombreux parasites agrégés, l'épithélium palléal engendre des villosités extrêmement importantes et ainsi, l'épaisseur totale du manteau se trouve considérablement augmentée. Petit à petit, les métacercaires vont se trouver enfouies à l'intérieur des profondes alvéoles puis complètement circonscrites (P. Bartoli, 1981). Les sphérules ainsi formées, dans lesquelles les métacercaires demeurent prisonnières, sont progressivement repoussées vers le plan sagittal du Lamellibranche par les vésicules en cours d'édification. Ainsi, chez des hôtes âgés et très parasités, on observe finalement un grand nombre de sphérules plus ou moins volumineuses remplissant presque la totalité de la région médio-dorsale du Lamellibranche. Les vésicules engendrées par le lobe palléal droit rejoignent celles élaborées par le lobe palléal gauche. Les vésicules anciennement formées ne communiquent plus avec le milieu extrapalléal général ; les métacercaires, toujours vivantes, perdent leur localisation interpallium et conchas habituelle et deviennent donc des parasites internes.

Une réaction palléale aussi intense et notamment la perte de la communication entre les vésicules englobant les métacercaires et le milieu extrapalléal n'a pas été signalée dans les Donax vittatus des côtes du Boulonnais. R. P. Dollfus (1912) a observé en effet que "chez le Donax, la poche d'épithélium palléal communique toujours largement avec la cavité conchopalléale ".

\section{4 - Description.}

Chez Donax trunculus et D. semistriatus, et seulement chez ces hôtes intermédiaires, les métacercaires de Meiogymnophallus strigatus ont une double destinée. Dans le cas le plus fréquent, elles sont enveloppées progressivement par les proliférations palléales qui isolent ainsi les parasites. Dans le même individu-hôte, certaines 
métacercaires ne sont pas circonscrites et demeurent alors libres dans l'espace extrapalléal. Ces deux types de métacercaires ont des aspects très différents. Nous décrirons d'abord les formes libres; nous les comparerons ensuite à celles qui ont été circonscrites par le pallium.

\section{a - Les métacercaires non enveloppées par le manteau.}

\section{Dimensions.}

Les dimensions résultent des mensurations de 20 métacercaires montées en préparations.

Longueur du corps : 280-380 (328).

Largeur du corps : 224-286 (259).

Ventouse orale : 72-92 (82) × 86-100 (91).

Ventouse ventrale : 40-56 (49) × 48-58 (53).

Rapport ventousaire, VO/VV : 1,57-1,83 $(1,70)$.

Espace préacétabulaire : 152-228 (193).

Espace postacétabulaire : 72-100 (86).

Pharynx : 22-28 (24) $\times$ 26-32 (29).

Rapport ventouse orale-pharynx, VO/P : 2,92-4,00 $(3,46)$.

Esophage : 20-50 (30).

Testicules : $60-120(95) \times 24-44(34)$.

Ovaire : 40-80 (61) $\times 28-40(32)$.

Masse vitelline : 40-80 (64) $\times$ 26-50 (36).

Aspect général.

Les métacercaires non enveloppées par le manteau sont relativement transparentes; leur aspect général est clair (fig. 3).

\section{Tégument.}

Presque tout le corps est revêtu d'épines ; dans la région médioventrale, une aire glabre s'étend entre le pharynx et la ventouse ventrale.

\section{Glandes céphaliques.}

Les glandes céphaliques sont extrêmement nombreuses ; on peut difficilement en évaluer le nombre. Elles couvrent la totalité de la région antérieure du corps et s'étendent vers l'arrière jusqu'au niveau du pharynx (fig. 4).

\section{Ventouses.}

La ventouse orale est souvent subterminale ; elle est dépourvue d'auricules.

La ventouse ventrale est située à la limite des tiers moyen et postérieur de la longueur du corps. Les six terminaisons sensorielles du cycle acétabulaire externe sont bien distinctes. 


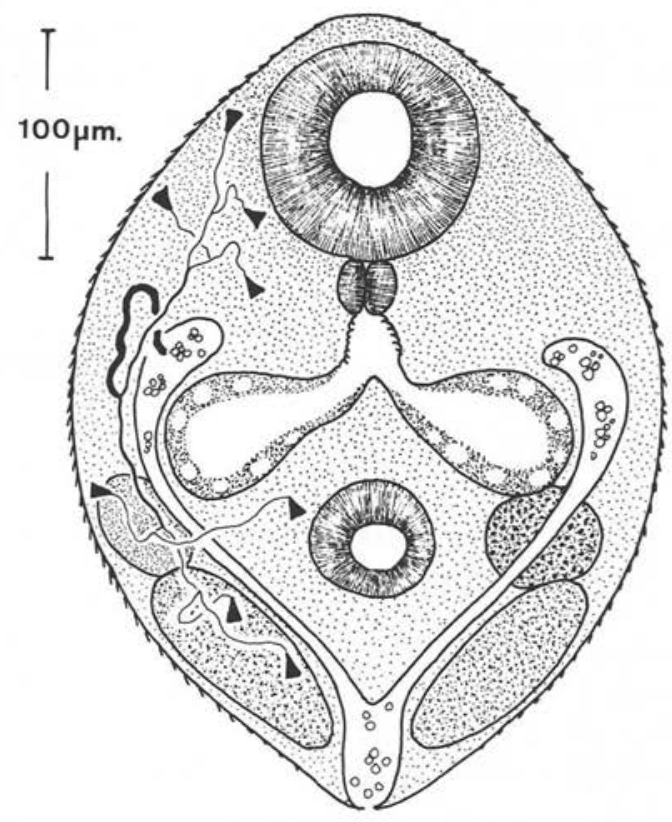

FIG. 3. - Meiogymnophallus strigatus (M. V. Lebour, I9o8) n. comb. Métacercaire libre, non enveloppée par les proliférations palléales. Deuxième hôte intermédiaire : Donax trunculus. Camargue. Morphologie générale et appareil excréteur.

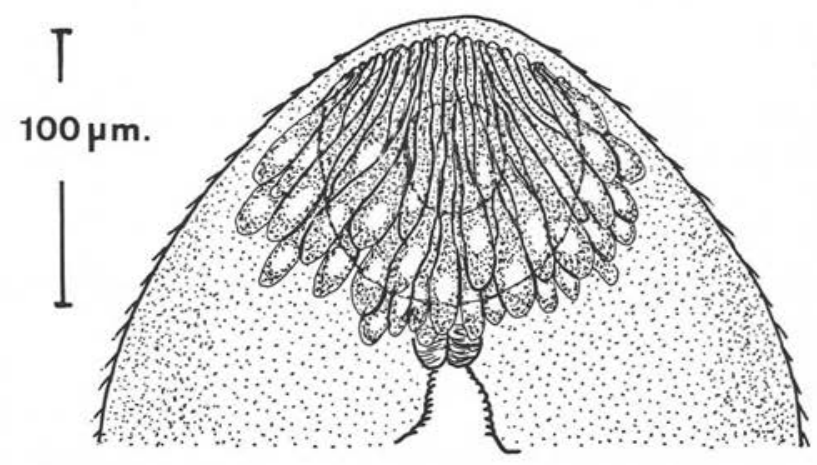

FIG. 4. - Meiogymnophallus strigatus (M. V. Lebour, I908)

n. comb. Métacercaire libre, parasite de Donax trunculus. Camargue. Glandes céphaliques.

\section{Appareil digestif.}

La bouche s'ouvre au centre de la ventouse orale. Le prépharynx est absent. Le pharynx est de taille réduite. Les caecums digestifs, extrêmement volumineux, sont constitués par des cellules dont le noyau est énorme; ils atteignent le niveau antérieur de la ventouse ventrale, plus rarement le niveau du centre acétabulaire. 


\section{Appareil génital mâle.}

Les testicules allongés, sont contenus presque totalement dans la région postacétabulaire; leur extrémité antérieure atteint habituellement le niveau du centre de la ventouse ventrale. Les testicules sont disposés près de la paroi du corps, leur grand axe parallèle à celle-ci. Une division transversale de la vésicule séminale a été parfois observée sur certains exemplaires. La pars prostatica est entourée de cellules prostatiques peu développées; celles-ci ne se jettent pas dans l'atrium génital (fig. 5). Le canal éjaculateur est absent.

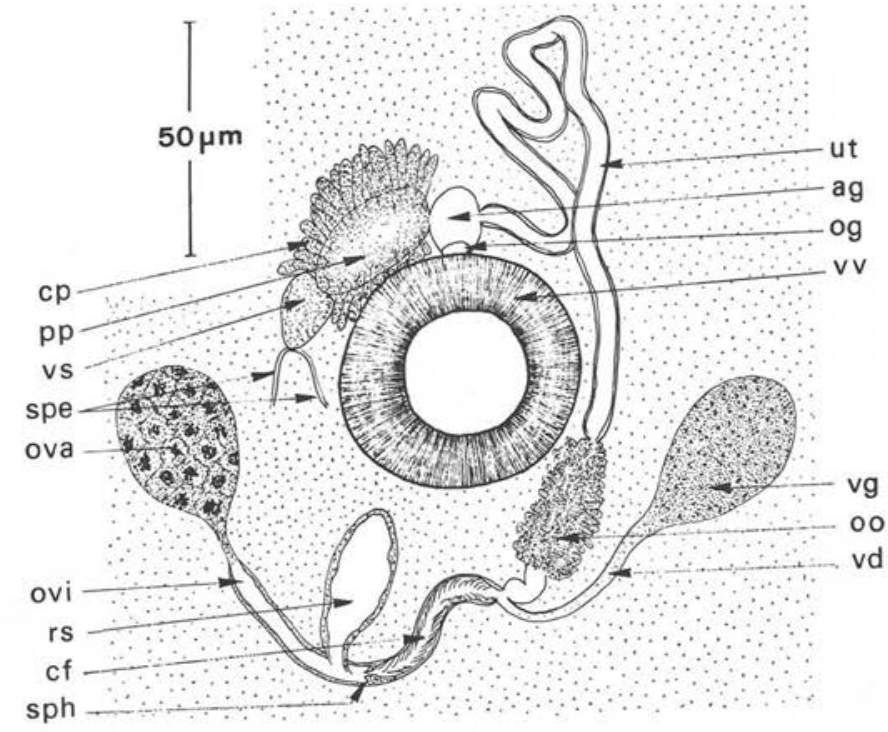

FIG. 5. - Meiogymnophallus strigatus (M. V. Lebour, I908) n. comb. Métacercaire libre, non enveloppée par le manteau. Deuxième hôte intermédiaire : Donax trunculus. Camargue. Appareil génital. ag : atrium génital ; $\mathrm{cf}$ : chambre de fécondation ; $\mathrm{cp}$ : cellules prostatiques ; og : orifice génital ; oo : ootype ; ova : ovaire ; ovi : oviducte ; pp : pars prostatica ; rs : réceptacle séminal ; spe : spermiductes; $s p h$ : sphincter ; ut : utérus ; vd : vitelloducte : vg : glande vitelline; vs : vésicule séminale; vv : ventouse ventrale.

\section{Appareil génital femelle.}

L'ovaire, de forme ovoïde, est localisé tantôt dans le côté droit du corps, tantôt dans le côté gauche. Disposé au niveau de la ventouse ventrale, il entre en contact par son extrémité postérieure, avec le testicule situé du même côté que lui. L'oviducte est long ; sa paroi est épaissie dans sa partie distale. Le réceptacle séminal, très volumineux, est toujours présent (fig. 5). Le canal de Laurer est absent. La chambre de fécondation est ciliée sur toute sa longueur ; à son extrémité débouche le vitelloducte. On observe une seule masse vitelline, plus ou moins ovoïde, du côté opposé à l'ovaire. L'ootype est nettement visible. L'utérus remonte en direction préacétabulaire en passant entre la ventouse ventrale et le testicule opposé à l'ovaire ; après avoir décrit quelques circonvolutions dans l'espace intercaecal, il débouche dans l'atrium génital. 
Atrium génital.

L'atrium génital, sphérique, est assez volumineux (fig. 5). Le pore génital est relativement grand; il est disposé au contact du bord antérieur de la ventouse ventrale, sur la ligne médio-ventrale. L'orifice génital n'est pas entouré de fibres musculaires ; on n'observe aucune dépression de la paroi ventrale du corps dans la région préacétabulaire.

\section{Appareil excréteur.}

L'appareil excréteur est composé de 16 protonéphridies; elles sont réparties entre elles comme l'indique la formule : $2[(2+2)+(2+2)]=16$ (fig. 3). Le premier groupe de cellules à flamme vibratile est localisé dans la région de la ventouse orale ; le second groupe est disposé au niveau et en arrière de la ventouse ventrale. Le canal collecteur principal est cilié ; il décrit une boucle avant de pénétrer vers l'extrémité des branches de la vessie, au niveau du bord antérieur des caecums digestifs. La vessie est constituée par un tronc impair court et par deux branches latérales. Les branches vésicales longent la face interne des testicules, s'immiscent entre les caecums digestifs et, selon le côté, l'ovaire ou la masse vitelline; elles s'incurvent ensuite puis s'étendent jusqu'au niveau du pharynx. La vessie est pleine de petites granulations sphériques de couleur noire. Le pore excréteur est terminal.
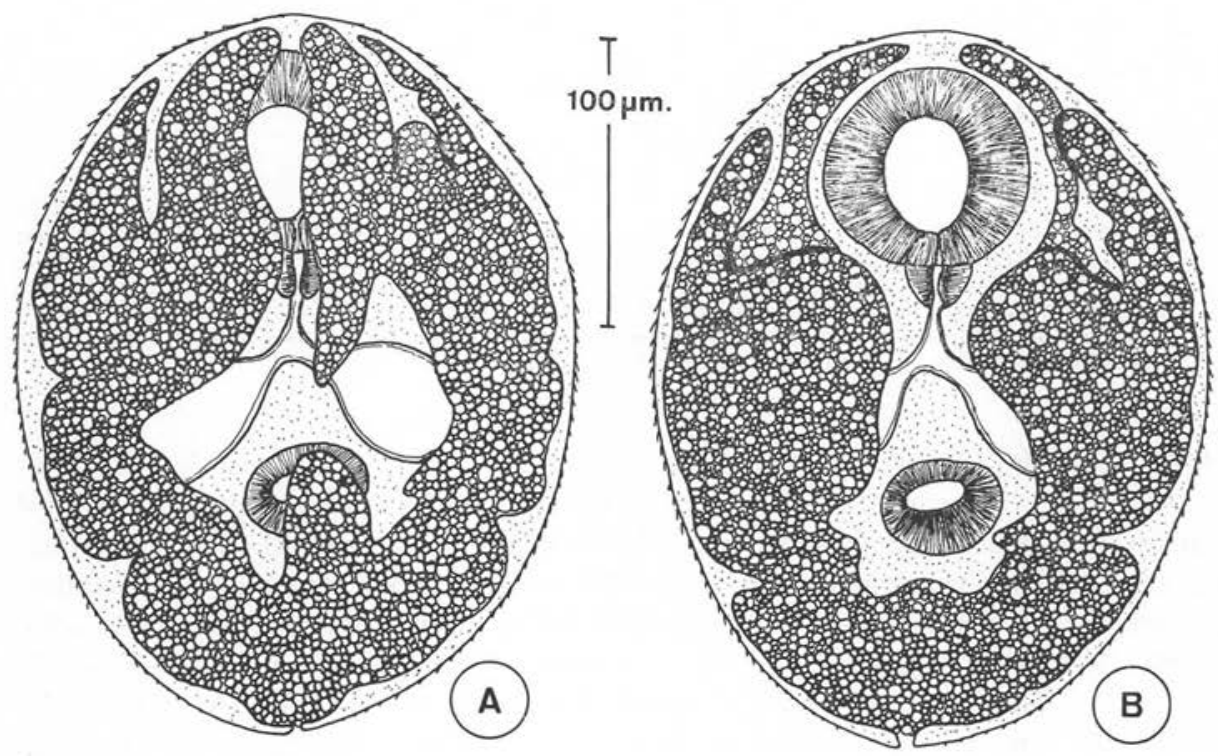

Fig. 6. - Meiogymnophallus strigatus (M. V. Lebour, I9o8) n. comb. Métacercaires enveloppées par le manteau de l'hôte. Deuxième hôte intermédiaire : Donax trunculus. Camargue. Morphologie générale. A : vue dorsale; B : vue ventrale. 


\section{b - Les métacercaires enveloppées par le manteau.}

Lorsque les métacercaires de Meiogymnophallus strigatus sont circonscrites par des proliférations palléales, leur aspect général, leur couleur en particulier, est très différent de celui des larves vivant librement entre la coquille et le manteau (fig. 6). En effet, nous observons une hypertrophie de la vessie excrétrice qui tend à remplir tout le volume du corps; elle est bourrée d'un nombre considérable de granulations noires atteignant jusqu'à $5 \mu \mathrm{m}$ de diamètre. La vessie est extrêmement boursoufflée ; comme l'a montré G. Rees (1939), l'extrémité de chaque branche, dans la région orale, est divisée en deux lobes par une profonde échancrure. La couleur générale de la métacercaire est d'un noir intense ; elle ressemble alors étrangement, aux dimensions près, à la métacercaire de Meiogymnophallus minutus (T. S. Cobbold, 1859) (syn. : Gymnophallus fossarum P. Bartoli, 1965) parasite de Cerastoderma glaucum et de divers autres Lamellibranches (P. Bartoli, 1972).

Les mensurations des métacercaires libres et enveloppées laissent entrevoir des différences importantes entre ces deux formes (tableau I). Par ailleurs, les larves non circonscrites par les expansions palléales ont une maturité génitale plus avancée que les autres.

TABLEAU I. - Meiogymnophallus strigatus (M. V. Lebour, 1908) n. comb. Dimensions comparées des métacercaires vivant librement dans l'espace extrapalléal de Donax trunculus et de celles enveloppées par le manteau du Lamellibranche. Camargue.

\begin{tabular}{lcc}
\hline & Métacercaires libres & Métacercaires enveloppées \\
\hline Longueur & $280-380(328)$ & $230-300(258)$ \\
Largeur & $224-286(259)$ & $174-217(195)$ \\
Espace préacétabulaire & $152-228(193)$ & $130-186(154)$ \\
Espace postacétabulaire & $72-100(86)$ & $59-90(69)$ \\
Ventouse orale (VO) & $72-92(82) \times 86-100(91)$ & $65-80(73) \times 68-83(75)$ \\
Ventouse ventrale (VV) & $40-56(49) \times 48-58(53)$ & $34-43(37) \times 37-45(40)$ \\
Pharynx (P) & $22-28(24) \times 26-32(29)$ & $19-25(21) \times 19-25(22)$ \\
VO/VV & $1,57-1,83(1,70)$ & $1,80-2,11(1,95)$ \\
VO/P & $2,92-4(3,46)$ & $3,1-3,9(3,55)$ \\
\hline
\end{tabular}

\section{IV. - Résultats expérimentaux}

Nous avons infesté artificiellement plusieurs Donax trunculus indemnes avec les cercaires émises par Tellina temuis; nous avons alors obtenu un grand nombre de très jeunes métacercaires. Malheureusement, la conservation de $D$. trunculus en aquarium est toujours difficile. Néanmoins, nous avons pu suivre l'évolution progressive des métacercaires pendant les dix-huit premiers jours. 
Six jours après le début de la contamination expérimentale, les jeunes métacercaires possèdent encore leurs deux paires de volumineuses glandes de pénétration ( fig. $7 \mathrm{~A}$ ) ; cependant, chez quelques individus, les corps cellulaires ont déjà disparu et il ne reste plus que les deux paires de canalicules et les pores au niveau desquels ils débouchent à l'extérieur. Quelques protonéphridies sont maintenant visibles. L'appareil génital s'ébauche : testicules, vésicule séminale, ovaire et utérus peuvent être distingués.

Dix-huit jours après l'infestation expérimentale, les métacercaires sont toujours libres. Les corps cellulaires et les conduits des deux paires de glandes de pénétration ont disparu; chez quelques individus on observe encore, en avant de l'orifice buccal, quatre petits points, restes des pores au niveau desquels les glandes débouchaient à l'extérieur ( $f i g .7$ B). La vessie excrétrice, agrandie, renferme de nombreuses petites granulations ; on aperçoit déjà l'ébauche d'une division à l'extrémité de ses branches. Dans la région postérieure de la vessie, de part et d'autre du plan sagittal, deux diverticules sont bien visibles. La formule excrétrice a pu être établie après avoir considéré un grand nombre de jeunes métacercaires ; elle est composée de 16 protonéphridies disposées selon la formule : $2[(2+2)+(2+2)]$.
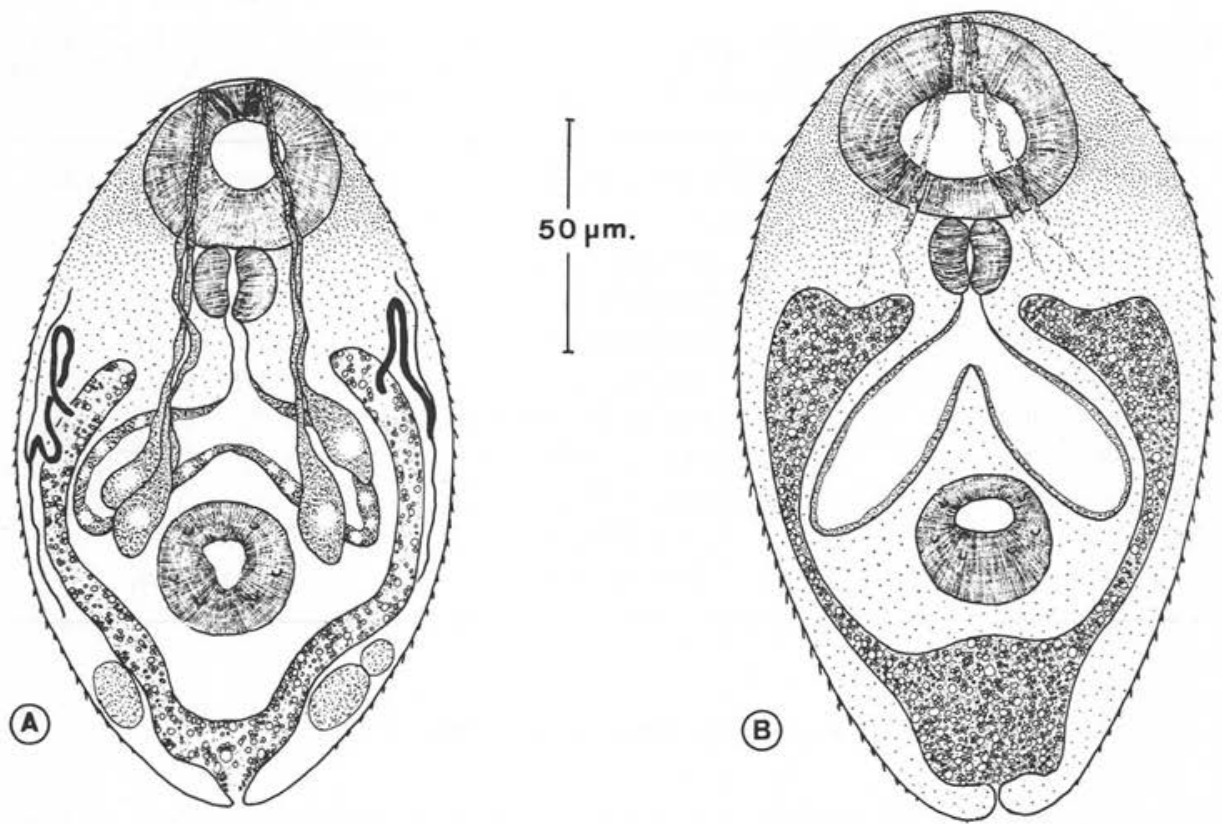

FIG. 7: - Meiogymnophallus strigatus (M. V. Lebour, I908) n. comb. Jeunes métacercaires développées expérimentalement chez Donax trunculus indemnes (deuxième hôte intermédiaire). Les cercaires proviennent de Tellina tenuis (premier hôte intermédiaire). Camargue. A : métacercaire âgée de six jours (vue dorsale) ; B : métacercaire âgée de r 8 jours (vue dorsale). 
Nous donnons dans le tableau II les dimensions de 10 métacercaires âgées de six jours et de dix autres âgées de dix-huit jours (individus fixés et montés entre lame et lamelle). Ce tableau met en évidence une particularité importante : la croissance de la ventouse orale diffère de celle de la ventouse ventrale. Le diamètre de la ventouse orale s'élève beaucoup plus rapidement que celui de la ventouse ventrale. Il en résulte que le rapport ventousaire se modifie sans cesse, passant progressivement d'une valeur moyenne de 1,23 chez la cercaire à une valeur moyenne de 1,70 chez la métacercaire âgée.

TABleaU II. - Meiogymnophallus strigatus (M. V. Lebour, 1908) n. comb. Dimensions comparées des cercaires émises par Tellina tenuis et des métacercaires âgées de 6 jours et de 18 jours développées expérimentalement chez Donax trunculus indemne. Camargue.

\begin{tabular}{|c|c|c|c|c|c|c|}
\hline \multirow[b]{2}{*}{ Longueur } & \multicolumn{2}{|c|}{ Cercaire } & \multicolumn{2}{|c|}{$\begin{array}{l}\text { Métacercaire } \\
\text { (6 jours) }\end{array}$} & \multicolumn{2}{|c|}{$\begin{array}{l}\text { Métacercaire } \\
\text { (18 jours) }\end{array}$} \\
\hline & $112-150$ & $(136)$ & $133-165$ & $(145)$ & $136-180$ & $(163)$ \\
\hline Ventouse orale (VO) & $30-38$ & $(34)$ & 31-39 & $(34)$ & $40-50$ & $(45)$ \\
\hline $\begin{array}{l}\text { Ventouse } \\
\quad \text { ventrale (VV) }\end{array}$ & $20-32$ & (28) & $22-31$ & $(27)$ & $26-32$ & (29) \\
\hline Pharynx $(\mathrm{P})$ & $13-17$ & (15) & $14-17$ & (16) & $15-20$ & (19) \\
\hline $\mathrm{VO} / \mathrm{VV}$ & $1,07-1,50$ & $(1,23)$ & $1,16-1,46$ & $(1,30)$ & $1,32-1,92$ & $(1,59)$ \\
\hline $\mathrm{VO} / \mathrm{P}$ & $1,88-2,57$ & $(2,28)$ & $2-2,40$ & $(2,19)$ & $2,15-2,60$ & $(2,42)$ \\
\hline
\end{tabular}

Les tentatives pour obtenir expérimentalement la forme adulte correspondant à cette métacercaire se sont toujours soldées par un échec. Les contaminations artificielles ont été pourtant tentées à diverses reprises sur plusieurs Oiseaux de différentes espèces : Larus argentatus, Aythya ferina, Aythya fuligula, Tadorna tadorna et Anas platyrhynchos.

\section{V. - Discussion}

Nous considèrerons successivement le cas du stade adulte puis celui des divers stades larvaires.

\section{1 - Le stade adulte.}

Plusieurs tentatives ont été faites pour rattacher Cercaria strigata à tel ou tel adulte connu. A. Giard (1907) trouve une concordance "en tous points " avec Gymnophallus somateriae G. Levinsen, 1881, une espèce parasite de l'intestin de Somateria mollissima (Eider). En 1908, M. V. Lebour suggère que l'hôte définitif soit plutôt recherché parmi les Laridés et non les Anatidés. Plus tard, R. P. Dollfus (1912) rapporte cette forme à une variété de G. somateriae : "Gymnophallus somateriae 
G. Levinsen, 1881, var. strigatus M. V. Lebour sp. ». Enfin G. Rees (1939) rapproche Cercaria strigata de G. macroporus H. L. Jameson et W. Nicoll, 1913 ou mieux de G. deliciosus (P. Olsson, 1893) T. Odhner, 1900.

Nous n'avons pas pu obtenir le stade adulte de Meiogymnophallus strigatus, ni par la voie expérimentale, ni dans la nature car les Anatidés purement marins fréquentant la Camargue sont rarissimes. Toutefois, la plupart des caractéristiques de la métacercaire nous permettent de classer cette espèce dans le genre Meiogymnophallus. Les particularités de ce genre ont été précisées successivement par B. L. James (1964) et par H. L. Ching (1965). Les seules divergences que l'on puisse souligner sont relatives aux glandes vitellines qui sont paires dans le genre Meiogymnophallus alors qu'ici, chez la métacercaire, il s'agit d'une masse unique. Il n'est pas impossible que cette masse vitelline puisse plus ou moins se diviser chez l'adulte. Les espèces du genre Meiogymnophallus sont des parasites intestinaux. On ignore encore la nature du microhabitat de l'adulte de $M$. strigatus.

Meiogymnophallus strigatus ressemble beaucoup à Meiogymnophallus jamesoni E. A. Bowers, 1965. Toutefois, une différence importante doit être soulignée. Chez $M$. jamesoni la vésicule séminale est indivise alors que chez $M$. strigatus une division transversale a été parfois observée chez certaines métacercaires. Il est possible que cette cloison n'ait pas été remarquée comme ce fut le cas pour Meiogymnophallus minutus (T. S. Cobbold, 1859). En effet E. A. Bowers et B. L. James (1967) ont décrit chez $M$. mimutus une vésicule séminale indivise ; elle était en fait bipartite (A. Sannia, 1977). Sur le plan écologique, nos recherches nous permettent d'affirmer que, en Camargue, l'hôte définitif de $M$. strigatus est un Anatidé marin, vraisemblablement Melanitta nigra et $M$. fusca. Il semble donc que $M$. jamesoni pourrait être suspecté comme étant la forme adulte probable des métacercaires de $M$. strigatus. Si cette supposition s'avérait exacte, $M$. jamesoni tomberait en synonymie avec $M$. strigatus.

\section{2 - Le stade métacercaire.}

Les métacercaires des Donacidés de Camargue ressemblent trop à Cercaria strigata pour que nous les considérions comme distinctes. Toutefois, une différence ne saurait passer inaperçue. En Provence, Tellina tenuis ne joue pas le rôle de deuxième hôte intermédiaire; par contre, sur les côtes de la Manche, plusieurs espèces de Tellines sont parasitées par les métacercaires : Tellina tenuis, $T$. fabula et $T$. solidula.

Sur les côtes de la Manche, Venerupis (= Tapes) decussata n'a jamais été signalé comme un deuxième hôte pour les métacercaires de Meiogymnophallus strigatus. De même, sur les côtes de Camargue, nous n'avons jamais rencontré une seule métacercaire de ce Gymnophallide dans les très nombreux $V$. decussata examinés. Par contre A. Palombi (1934) signale dans ces Lamellibranches de la baie de Naples, des métacercaires qu'il appelle Metacercaria (Gymnophallus) strigata. En raison de la nature du deuxième hôte, et en s'appuyant sur la description donnée par A. Palombi, nous rattachons ces métacercaires à Meiogymnophallus minutus (T. S. Cobbold, 1859) (syn : Gymnophallus fossarum P. Bartoli, 1965). Il apparaît ainsi que $M$. strigatus est signalé pour la première fois en Méditerranée. 


\section{3 - Le stade cercaire.}

A notre connaissance, c'est la première fois qu'une furcocercaire est signalée dans Tellina tenuis. Nous précisons que Cercaria dichotoma M. V. Lebour, 1908 (nec J. Müller in de la Valette Saint Georges, 1855) est issue de Scrobicularia tenuis ; ce Lamellibranche, que par ailleurs M. V. Lebour distingue de Tellina tenuis, appartient en fait au genre Abra.

P. Pelseneer (1906) a été l'un des premiers à soupçonner le lien existant entre les métacercaires des Gymnophallidae et les furcocercaires; il voyait une correspondance entre les métacercaires de Donax et de Tellina et une cercaire que l'on trouve au même. endroit dans Syndosmya (= Abra) alba : Cercaria syndosmyae P. Pelseneer, 1906. Mais cette éventualité fut immédiatement repoussée par M. V. Lebour (1908) sur le seul examen de la morphologie de la cercaire. Cet auteur pensait que Cercaria syndosmyae, dont les ventouses sont subégales, ne pouvait correspondre à une métacercaire dont le rapport ventousaire est plus élevé ; de plus M. V. Lebour rejetait l'idée que les cercaires des Gymnophallidae aient une queue : "they never have tails ». En 1939, G. Rees affirme avoir trouvé les cercaires correspondant à "Cercaria " strigata dans Cerastoderma (= Cardium) edule : " the sporocysts occur deep down in the digestive gland. They are thin-walled colourless oval structures filled with cercariae in various stages of development ". Malheureusement l'auteur ne donne aucune description des cercaires ni aucune preuve permettant de les rattacher à Cercaria strigata. Aussi H. W. Stunkard et J. R. Uzmann (1958) déclaraient : " the cercariae were not described other than the statement that they were the same as the metacercariae ". Tout laisse supposer que les cercaires trouvées par G. Rees dans $C$. edule ne sont pas des cercaires de Gymnophallidae ; en effet cet auteur était persuadé, comme M. V. Lebour, que " no tailed forms of Gymnophallus larvae are known ». Rappelons toutefois que $C$. edule est parasité par les furcocercaires de Gymnophallus choledochus T. Odhner, 1900 dont le cycle biologique a été élucidé par B. Loos-Frank (1969).

\section{BIBLIOGRAPHIE}

Bartoli P. : Les cycles biologiques de Gymnophallus nereicola J. Rebecq et G. Prévot, 1962 et G. fossarum P. Bartoli, I965, espèces jumelles parasites d'Oiseaux de rivages marins (Trematoda, Digenea, Gymnophallidae). Ann. Parasitol. Hum. Comp., 1972, 47, 193-223.

Bartoli P. : Démographie et phénomène de compétition intraspécifique des Gymnophallidae Morozov, 1955 chez les deuxièmes hôtes intermédiaires. Ann. Parasitol. Hum. Comp., I981, $56,33-44$.

Bowers E. A. : A description of Meiogymnophallus jamesoni sp. nov. (Trematoda : Gymnophallidae) from the intestine of the common scoter, Melanitta nigra L. Ann. Magazine Natural History, 1965 , ser. $13,8,277-283$.

Bowers E. A., JAmes B. L. : Studies on the morphology, ecology and life-cycle of Meiogymnophallus minutus (Cobbold, I859) comb. nov. (Trematoda : Gymnophallidae). Parasitology, G.B., $1967,57,28 \mathrm{I}-300$.

ChIng H. L. : Life cycles of Lacunovermis conspicuus n. gen., n. sp. and Meiogymnophallus multigemmulus n. gen., n. sp. (Gymnophallidae : Trematoda) from Macoma inconspicua and Diving Ducks from Vancouver, Canada. Proc. Helminthol. Soc. Wash., 1965, 32, 53-63.

Dollfus R. P. : Une métacercaire margaritigène parasite de Donax vittatus Da Costa. Contribution à l'étude des Trématodes marins du Boulonnais. Mem. Soc. Zool. France, 1912, 24, 85-I44. 
Giard A. : Sur un Distome (Brachycoelium sp.) parasite de Pélécypodes. C.R. Soc. Biol. Paris, I897, 49, 956-957.

GIARD A. : Sur les Trématodes margaritigènes du Pas-de-Calais (Gymnophallus somateriae Levinsen et G. bursicola Odhner). C.R. Soc. Biol. Paris, 1907, 63, 416-420.

James B. L. : The life-cycle of Parvatrema homoeotecnum sp. nov. (Trematoda : Digenea) and a review of the family Gymnophallidae Morozov, I955. Parasitology, G.B., I964, 54, I-4I.

Lebour M. V. : Trematodes of the Northumberland coast, no II. Trans. Nat. Hist. Soc. Northumberland, 1908, 3, 28-45.

Loos-Frank B. : Zur kenntnis der Gymnophalliden Trematoden des Nordseeraumes. I. Die Alternativ-Zyclen von Gymnophallus choledochus Odhner, 1900. Z. Parasitenk., I969, 32, I 35 -I 56.

PALOmbi A. : Gli stadi larvali dei Trematodi del Golfo di Napoli. Io Contributo allo studio della morfologia, biologia e sistematica delle cercarie marine. Publ. Staz. Zool. Napoli, 1934, I4, 5I-94.

Pelseneer P. : Trématodes parasites de Mollusques marins. Bull. Sc. France Belgique, 1906, s, I6I-I 86.

Peres J. M., Picard J. : Nouveau manuel de bionomie benthique de la Mer Méditerranée. Rec. Trav. St. Mar. End., I964, 31, 5-137.

Rees G. : Cercaria strigata Lebour from Cardium edule and Tellina tenuis. Parasitology, G.B., I939, $31,45^{8-463}$.

Sannia A. : Digenea from marine Molluscs. Ph. D. Thesis, I977, University of Wales.

Stunkard H. W., Uzmann J. R. : Studies on digenetic Trematodes of the genera Gymnophallus and Parvatrema. Biol. Bull., 1958, 115, 276-302. 